\title{
Reanastomose Tubária Laparoscópica: Resultados Preliminares
}

\author{
Laparoscopic Tubal Anastomosis: Preliminary Results
}

\begin{abstract}
Sérgio Conti Ribeiro, Renata Assef Tormena, Alessandra de Araújo Silva Bedin
Cassiana Rosa Galvão Giribela, Carlos Roberto Izzo, Nelson da Cruz Santos, José Aristodemo Pinotti
\end{abstract}

\section{RESUM0}

Objetivos: avaliar as taxas de gravidez após realização de reversão laparoscópica de obstrução tubária por laqueadura prévia.

Métodos: entre dezembro de 1998 e dezembro de 2001, realizamos estudo prospectivo no qual foram incluidas 26 pacientes previamente submetidas a esterilização tubária. As pacientes tinham idades entre 28 e 37 anos e a reanastomose foi efetuada por via laparoscópica. $O$ periodo de seguimento variou entre 150 e 425 dias e as taxas de permeabilidade tubária (estudada por meio da histerossalpingografia) e as taxas de gestação foram avaliadas.

Resultados: a reversão laparoscópica pôde ser realizada em 23 pacientes, sendo bilateral em 21. Duas pacientes apresentavam uma tuba extremamente danificada e três haviam sido submetidas a fimbriectomia bilateral. O tempo cirúrgico variou de 95 a 155 minutos e a alta hospitalar ocorreu na manhã seguinte em todos os casos. Constatou-se permeabilidade tubária em 15 pacientes (15/23) e a taxa de gestação foi 56,5\% (13/23), sem gestações ectópicas. $O$ intervalo entre a interrupção do uso do condom e a gravidez foi de até quatro meses em nove pacientes e de até 10 meses em quatro pacientes.

Conclusão: em casos selecionados, a reversão tubária videolaparoscópica pode ser oferecida à pacientes laqueadas que desejam novas gestações. A seleção adequada das pacientes, assim como técnica cirúrgica meticulosa, são os fatores-chave para atingir taxas de gestação satisfatórias.

PALAVRAS-CHAVE: Reanastomose tubária. Infertilidade. Contracepção cirúrgica. Laqueadura tubária.

\section{Introdução}

Considerado questão de saúde pública em todo o mundo, o planejamento familiar recebe atenção da comunidade médica tanto em países desenvolvidos quanto naqueles em desenvolvimento. Diferentes métodos contraceptivos estão disponiveis, porém a esterilização tubária ainda é um dos mais utilizados, sendo realizada inclusive em mulheres com idade inferior a 30 anos, grupo que freqüentemente manifesta arrependimento posterior $^{1,2}$

A técnica de Pomeroy é a habitualmente

Clínica Ginecológica do Hospital das Clínicas da Faculdade de Medicina da USP

Correspondência:

Sérgio Conti Ribeiro

Rua Itambé, 289, apto 1207 - Higienópolis

01239-001 - São Paulo - SP

Tel / Fax. (11) 3168-5311

e-mail: SergioRibeiroGO@aol.com utilizada para a esterilização tubária, danificando aproximadamente $3 \mathrm{~cm}$ da porção istmo-ampolar da tuba. O anel de Sylastic ${ }^{\circledast}$ é opção fácil e foi muito utilizada para esterilização videolaparoscópica na década de 70 , destruindo $3 \mathrm{~cm}$ da porção ístmica (ou ampolar) da tuba. Atualmente, a técnica videolaparoscópica utiliza tanto coagulação com corrente bipolar quanto clipes de $4 \mathrm{~mm}$ de largura, danificando até $2 \mathrm{~cm}$ de cada tuba. Ocasionalmente pode ser necessária a utilização de múltiplos clipes ou coagulação bipolar para controlar sangramentos, aumentando a área lesada ${ }^{3,4}$.

Inúmeros motivos levam algumas mulheres esterilizadas a manifestar o desejo de nova gestação, entre os quais destacamos a mudança de cônjuge e a perda de um filho ${ }^{2}$. O procedimento padrão para estas pacientes é a recanalização das tubas pela reanastomose tubária microcirúrgica por laparotomia ${ }^{5,6}$. Em nosso meio, após a reversão de laqueadura verificaram-se taxas de 
permeabilidade e gestação de 89 e 56\%, respectivamente $^{7}$.

O desenvolvimento de novas técnicas e o aprimoramento dos instrumentos permitiram a abordagem laparoscópica para este procedimento, agregando os benefícios já conhecidos da via laparoscópica, entre os quais o caráter ambulatorial, menor trauma cirúrgico e retorno precoce às atividades habituais. Entretanto, os resultados iniciais em termos de permeabilidade tubária e gestações eram inferiores aos obtidos pela técnica microcirúrgica por laparotomia ${ }^{8-11}$.

Outra questão que deve ser colocada é se nos dias atuais ainda há espaço para a cirurgia tubária nas pacientes com desejo de reversão de laqueadura. Alguns autores recomendam a fertilização in vitro (FIV) como primeira alternativa, alegando que com a cirurgia é necessário internação e anestesia, além de ter que se esperar algum tempo, em média seis meses, até a paciente engravidar ${ }^{12,13}$. Por outro lado, o preço do tratamento e das medicações, o risco de hiperestimulação ovariana e de gestações múltiplas e a eventual necessidade de repetição da técnica devem ser considerados diante da indicação de FIV.

O objetivo deste trabalho é avaliar os resultados de permeabilidade tubária e taxas de gestação em pacientes submetidas a reanastomose tubária laparoscópica.

\section{Pacientes e Métodos}

Efetuamos estudo prospectivo no período de dezembro de 1998 a dezembro de 2001, no qual foram incluídas 26 mulheres com esterilização tubária bilateral. Todas as pacientes foram previamente orientadas em relação aos riscos da abordagem laparoscópica e assinaram consentimento informado de acordo com a Comissão de Ética do Hospital das Clínicas da FMUSP. A idade das pacientes variou de 28 a 37 anos (média 34 anos) e a esterilização tubária havia sido realizada pela técnica de Pomeroy ou variação desta (ligadura ou secção mediana tubária) durante a realização de parto cesárea. Em todas as pacientes a investigação laboratorial quanto à esterilidade foi normal, incluindo dosagem de FSH no $3^{\circ}$ dia do ciclo, espermograma, ultra-sonografia transvaginal, biópsia de endométrio e dosagem de progesterona e prolactina sérica no $19^{\circ}$ dia do ciclo. A histerossalpingografia sugeria obstrução ístmica bilateral em todas as pacientes. O tempo decorrido entre a esterilização tubária e a reanastomose variou de 1 a 12 anos.

O procedimento cirúrgico foi realizado com a paciente em posição semiginecológica sob anestesia geral e sempre utilizando cânula intrauterina para movimentação do útero e realização de cromotubagem. No momento da indução anestésica administrava-se $1 \mathrm{~g}$ de cefazolina $\left(\mathrm{Kefazol}^{\circledR}\right)$, dose repetida após 4 horas, seguindo orientação da Comissão de Infecção Hospitalar do Hospital das Clínicas da FMUSP. Após a inserção da agulha de Veress, realizava-se a insuflação peritoneal de $\mathrm{CO}_{2}$ até atingir a pressão intra-abdominal pré-estabelecida entre 25 e $30 \mathrm{mmHg}$, seguida da inserção de trocater umbilical de 10 mm e subseqüente redução da pressão para 12 $\mathrm{mmHg}^{14}$. A fase inicial do procedimento consistia em avaliar se a reversão tubária seria tecnicamente possível, de acordo com o comprimento da tuba, qualidade da fïmbria e eventuais aderências. Quando o comprimento tubário era menor que 4 $\mathrm{cm}$, na presença de aglutinação das fímbrias ou mesmo ausência das mesmas (fimbriectomia) ou em casos de processo aderencial intenso envolvendo as trompas, o procedimento era encerrado e a paciente encaminhada para o programa de FIV. Quando a situação era favorável, três trocateres auxiliares de $5 \mathrm{~mm}$ eram inseridos na região inferior do abdome para a passagem dos demais instrumentos.

A reanastomose tubária incluía 4 etapas principais: i) identificação e remoção da área danificada da tuba (granuloma), delineada após a injeção de azul de metileno através da cânula intra-uterina (Valtchev $\left.{ }^{\circledR}\right)$; ii) verificação da permeabilidade do coto distal por meio da cateterização e instilação de azul de metileno, utilizando cateter de anestesia peridural; iii) aproximação dos cotos utilizando ponto de Vicryl $^{\oplus}$ 5-0 ou Mononylon ${ }^{\circledR}$ 5-0, aplicado no mesossalpinge, permitindo alinhamento perfeito dos dois lumens tubários, e iv) anastomose tubária propriamente dita, realizada em plano único, abrangendo serosa e muscular, utilizando 4 pontos de Mononylon ${ }^{\circledR} 6$ 0 aplicados nos pontos cardinais da tuba.

Após a cirurgia os casais eram orientados para utilizar preservativo masculino durante 60 dias após a cirurgia. A permeabilidade tubária era avaliada por meio da histerossalpingografia três meses após a cirurgia.

\section{Resultados}

A reanastomose tubária laparoscópica foi tecnicamente possivel em vinte e três pacientes, das quais foi bilateral em vinte e uma. Três mulheres haviam sido submetidas a fimbriectomia bilateral. Em duas pacientes a reversão foi reali- 
zada em apenas uma tuba, devido às condições inadequadas da outra tuba. O tempo cirúrgico variou entre 95 e 155 minutos. Todas as pacientes receberam alta na manhã seguinte à cirurgia e não ocorreu qualquer tipo de complicação significativa.

O acompanhamento posterior variou de 150 a 425 dias (média de 252 dias). A permeabilidade tubária, avaliada pela histerossalpingografia três meses após a cirurgia, foi constatada em 15 pacientes (15/23), incluindo uma daquelas com reanastomose unilateral. A taxa de gravidez foi de $56,5 \%(13 / 23)$, sem gestações ectópicas. Uma das gestações evoluiu para abortamento com oito semanas de idade gestacional (1/13). Entre as pacientes que engravidaram, o intervalo decorrido entre a interrupção do uso do condom e a gravidez foi de até quatro meses em nove pacientes e de até 10 meses em quatro pacientes.

\section{Discussão}

Nas últimas três décadas a reanastomose microcirúrgica tem sido realizada para a reversão de esterilização tubária naquelas pacientes que manifestam arrependimento e interesse por novas gestações ${ }^{5}$. Alguns fatores interferem na taxa de gestações nas pacientes submetidas a reanastomose tubária. O comprimento da tuba após a reanastomose é o principal fator prognóstico, com resultados pobres quando o comprimento tubário é menor que $4 \mathrm{~cm}$. Além disso, quanto mais curta a tuba, maior o tempo para a gestação ocorrer ${ }^{4-6}$. Em nosso estudo, a reversão cirúrgica não era realizada quando o comprimento tubário era menor que $4 \mathrm{~cm}$, o que ocorreu em uma paciente (uma tuba). Outra paciente apresentava fímbrias aglutinadas em uma tuba, não sendo possivel obter a permeabilidade do coto distal, mesmo com a passagem do cateter de peridural e injeção de azul de metileno sob alta pressão.

Em relação à técnica empregada para esterilização tubária, a utilização de clipes na região istmica parece proporcionar melhores resultados de permeabilidade tubária após as tentativas de reanastomose tubária ${ }^{4-6}$. Na nossa casuística foram incluídas apenas pacientes que haviam sido submetidas à esterilização tubária durante parto cesáreo e em nenhum dos casos havia sinais sugestivos da utilização de clipes metálicos.

O desenvolvimento de técnicas videolaparoscópicas gerou o interesse de realizar a reanastomose tubária utilizando o acesso laparoscópico. Utilizando cola biológica e um cateter como guia para permitir o acoplamento dos dois cotos tubários, a primeira gravidez intra-uterina após reversão laparoscópica de laqueadura foi descrita em $1989^{8}$. Apesar dessa abordagem parecer mais atraente, os resultados iniciais de taxas de fertilidade e de gestação eram inferiores àqueles relatados após reversão por laparotomia ${ }^{9-11}$.

O surgimento de pinças de 2 e $3 \mathrm{~mm}$ de diâmetro, de câmeras de 3 chips e de monitores de alta resolução, associados ao aperfeiçoamento técnico-cirúrgico, permitiu a obtenção de resultados comparáveis à microcirurgia por laparotomia. Bissonnette et al. ${ }^{15}$ realizaram estudo prospectivo com 102 pacientes submetidas à reanastomose tubária videolaparoscópica, utilizando apenas um ponto para realizar a anastomose. A vantagem desta técnica era o menor tempo operatório. Em oito pacientes constatou-se obstrução tubária bilateral na histerossalpingografia pós-operatória; cinco apresentaram gestações ectópicas $(7,2 \%) \mathrm{e}$ 69 pacientes $(70 \%)$ tiveram gestações intra-uterinas. Posteriormente, outros autores, realizando o procedimento de forma idêntica à técnica microcirúrgica por laparotomia (sutura da camada muscular da tuba com quatro pontos incluindo a mucosa, seguida por sutura intermitente da camada serosa), relataram taxas de permeabilidade e gestação semelhantes à laparotomia ${ }^{16}$.

Avaliando resultados de fertilidade, Cha et al. ${ }^{17}$ verificaram taxas de gestação de 80,5 e $80 \%$ em 76 pacientes submetidas a reanastomose tubária por laparoscopia ou laparotomia, respectivamente.

Em nosso Serviço não contamos com instrumentos de 2 ou $3 \mathrm{~mm}$, nem com microcâmeras de 3 chips, sendo utilizado o equipamento laparoscópico convencional. Com tal equipamento é praticamente impossível realizar a sutura dos cotos em dois planos e preferimos realizá-la com quatro ou cinco pontos em plano seromuscular único. As limitações instrumentais, bem como a curva de aprendizagem, explicam, ao menos em parte, porque nossa taxa de gestação $(56,5 \%)$ é menor que a encontrada na literatura (70 a 80\%). Salientamos que a reanastomose tubária videolaparoscópica é procedimento difícil que requer treinamento específico, podendo ser enquadrada no nível IV de dificuldade da escala de Chapron et al. ${ }^{18}$. Por outro lado, a taxa de gestação em nossa casuística foi idêntica à relatada quando se realiza a reanastomose por laparotomia ${ }^{7}$.

Yoon et al. ${ }^{16}$ não verificaram diferença significativa nas taxas de gestação de acordo com o método de esterilização utilizado, com o local da reanastomose tubária, com o comprimento da trompa, ou quanto à presença de doenças pélvicas como miomas uterinos, endometriose ou aderências. Houve, sim, diferença nas taxas de gestação 
entre pacientes que foram submetidas a reanastomose uni e bilateral ${ }^{16}$. Em nosso estudo, selecionamos apenas pacientes com obstrução ístmica e a presença de aderências firmes envolvendo as trompas foi verificada em quatro pacientes, em duas das quais a reanastomose foi unilateral, conforme explicamos anteriormente. Até o presente momento, observou-se gestação apenas em uma dessas pacientes. Ressaltamos, porém, que a presente casuística é insuficiente para retirarmos qualquer conclusão significativa em relação a diferenças nas taxas de gestação nas pacientes com aderências associadas, bem como em relação à reanastomose uni ou bilateral.

Uma questão que deve ser colocada é se nos dias atuais ainda há espaço para a cirurgia tubária nas pacientes com desejo de reversão de laqueadura. Vários autores recomendam a FIV como primeira alternativa para todos os casais inférteis devido à presença de lesão tubária de qualquer natureza, sendo relatadas taxas de gestação de 21 a $46 \%$ com essa técnica ${ }^{12,13}$. Em publicação recente, a Sociedade Européia de Reprodução Humana e Embriologia (ESHRE) ${ }^{19}$ relata taxa média de gestação por ciclo próxima a $27 \%$ nas pacientes submetidas a FIV em 521 centros de fertilização espalhados por 18 países na Euro$\mathrm{pa}^{19}$. Nos Estados Unidos da América os resultados são pouco superiores, atingindo $36,5 \%$ de gestação por ciclos de FIV ${ }^{20}$.

Conforme discutimos anteriormente, os resultados atuais de gestação em pacientes submetidas a reanastomose tubária por laparotomia ou por laparoscopia são repetidamente superiores aos obtidos nas diversas técnicas de FIV. Além disso, permite a paciente engravidar mais de uma vez sem a necessidade de realizar qualquer outro procedimento. A desvantagem da cirurgia é a necessidade de internação e de se esperar algum tempo, em média seis meses, até a paciente engravidar e, muitas vezes, a ansiedade do casal não é administrada de forma adequada. Por outro lado, o preço do tratamento e das medicações, o risco de gestações múltiplas e a eventual necessidade de repetição da técnica devem ser considerados em face da indicação de FIV.

Parece-nos que em casais sem outros fatores de redução de fertilidade associados, a reanastomose tubária deve ser considerada como a opção adequada na busca de novas gestações. Alternativamente, pacientes com mau prognóstico ou idade mais avançada devem ser encaminhadas direto para programas de FIV. Tal posição é compartilhada mesmo em grandes centros de reprodução humana em países onde ambos os procedimentos são igualmente oferecidos e não há qualquer interesse econômico favorecendo esta ou aquela conduta ${ }^{21,22}$.

Concluímos que, em casos selecionados, a reversão tubária videolaparoscópica pode ser oferecida às pacientes laqueadas que desejam novas gestações. Seleção adequada das pacientes, técnica cirúrgica meticulosa e disponibilidade de equipamentos adequados são os fatores chave para atingir taxas de gestação satisfatórias. Pacientes com mau prognóstico cirúrgico, com fatores associados de redução de fertilidade ou idade mais avançada devem ser encaminhadas direto para programas de FIV.

\section{ABSTRACT}

Purpose: to evaluate pregnancy outcome after laparoscopic tubal anastomosis.

Method: from December 1998 to December 2001, 26 patients with bilateral tubal ligation who underwent laparoscopic tubal anastomosis were prospectively evaluated. Patients' age ranged from 28 to 37 years. The follow-up period ranged from 150 to 425 days and tubal patency (evaluated by hysterosalpingography) and pregnancy outcome were evaluated.

Results: laparoscopic tubal reversal was performed in 23 patients. Bilateral reversal was attempted in all except two patients. The operation time ranged from 95 to 155 minutes and all patients were discharged on the following morning. Tubal patency was confirmed in 15 patients $(15 / 23)$. Pregnancy rate was $56.5 \%$ (13/23), without ectopic pregnancies. Nine patients got pregnant in up to four months and four in up to nine months after discontinuing the use of condom.

Conclusion: in selected cases, laparoscopic tubal reversal can be performed in patients who had been submitted to tubal sterilization and desire new pregnancies. Patient selection as well as meticulous surgical technique are key factors in achieving satisfactory pregnancy rates.

KEY WORDS: Tubal anastomosis. Infertility. Surgical contraception. Tubal sterilization.

\section{Referências}

1. Fernandes AMS, Arruda MS, Palhares MAR, Benetti Junior ND, Moreira CM. Seguimento de mulheres laqueadas arrependidas em serviço público de esterilidade conjugal. Rev Bras Ginecol Obstet 2001; 23:69-73.

2. Ades A. Identificação de fatores associados ao arrependimento após laqueadura tubária [dissertação]. São Paulo: Universidade de São Paulo; 1996. 
3. Siegler AM, Hulka J, Peretz A. Reversibility of female sterilization. Fertil Steril 1985; 43:499-510.

4. Rock JA, Guzick DS, Katz E, Zacur HA, King TM. Tubal anastomosis: pregnancy success following reversal of Falope ring or monopolar cautery sterilization. Fertil Steril 1987; 48:13-7.

5. Gomel V. Microsurgical reversal of female sterilization: a reappraisal. Fertil Steril 1980; 33:58797.

6. Winston RM. Microsurgical tubocornual anastomosis for reversal of sterilization. Lancet $1977 ; 1: 284-5$.

7. Izzo VM, Srougi M, Salvatore CA, Souza AZ. Reversão microcirúrgica da esterilização tubária. Ginecol Obstet Bras 1984; 7:31-9.

8. Sedbon E, Delajolinieres JB, Boudouris O, Madelenat P. Tubal desterilization through exclusive laparoscopy. Hum Reprod 1989; 4:158-9.

9. Reich H, McGlynn F, Parente C, Sekel L, Levie M. Laparoscopic tubal anastomosis. J Am Assoc Gynecol Laparosc 1993; 1:16-29.

10.Katz E, Donesky BW. Laparoscopic tubal anastomosis. A pilot study. J Reprod Med 1994; 39:497-8.

11.Istre O, Olsboe F, Trolle B. Laparoscopic tubal anatomosis: reversal of sterilization. Acta Obstet Gynecol Scand 1993; 72:680-1.

12. Holst N, Maltau JM, Forsdahl F, Hansen LJ. Handling of tubal infertility after introduction of in vitro fertilization: changes and consequences. Fertil Steril 1991; 55: 140-3.

13. Chardon C, Boulieu D, Rochet Y, Payan F, Ayzac L. Stérilité d'origine tubaire: quel traitement proposer: FIV ou chirurgie? Rev Fr Gynécol Obstét 1992; 87:355-60.
14. Reich H, Ribeiro SC, Rasmussen C, Rosenberg J, Vidali A. High-pressure trocar insertion technique. JSLS 1999; 3:45-8.

15.Bissonnette F, Lapensée L, Bouzayen R. Outpatient laparoscopic tubal anastomosis and subsequent fertility. Fertil Steril 1999; 72:549-52.

16.Yoon TK, Sung HR, Kang HG, Cha SH, Lee CN, Cha KY. Laparoscopic tubal anastomosis: fertility outcome in 202 cases. Fertil Steril 1999; 72:1121-6.

17. Cha SH, Lee MH, Kim JH, Lee CN, Yoon TK, Cha KY. Fertility outcome after tubal anastomosis by laparoscopy and laparotomy. J Am Assoc Gynecol Laparosc 2001; 8:348-52.

18. Chapron C, Devroey P, Dubuisson JB, Pouly JL, Vercellini P. ESHRE guidelines for training, accreditation and monitoring in gynaecological endoscopy. Hum Reprod 1997; 12:867-8.

19.Nygren KG, Andersen AN. Assisted reproductive technology in Europe, 1998. Results generated from European registers by ESHRE. European Society of Human Reproduction and Embryology. Hum Reprod 2001; 16:2459-71.

20.ASRM/SART Registry. Assisted reproductive technology in the United States: results generated from the American Society for Reproductive Medicine/Society for Assisted Reproductive Technology Registry. Fertil Steril 2000; 74:641-54.

21.Penzias AS, DeCherney AH. Is there ever a role for tubal surgery? Am J Obstet Gynecol 1996; 174:121823.

22.Posaci C, Camus M, Osmanagaoglu K, Devroey P. Tubal surgery in the era of assisted reproductive technology: clinical options. Hum Reprod 1999; 14 Suppl 1:120-36.

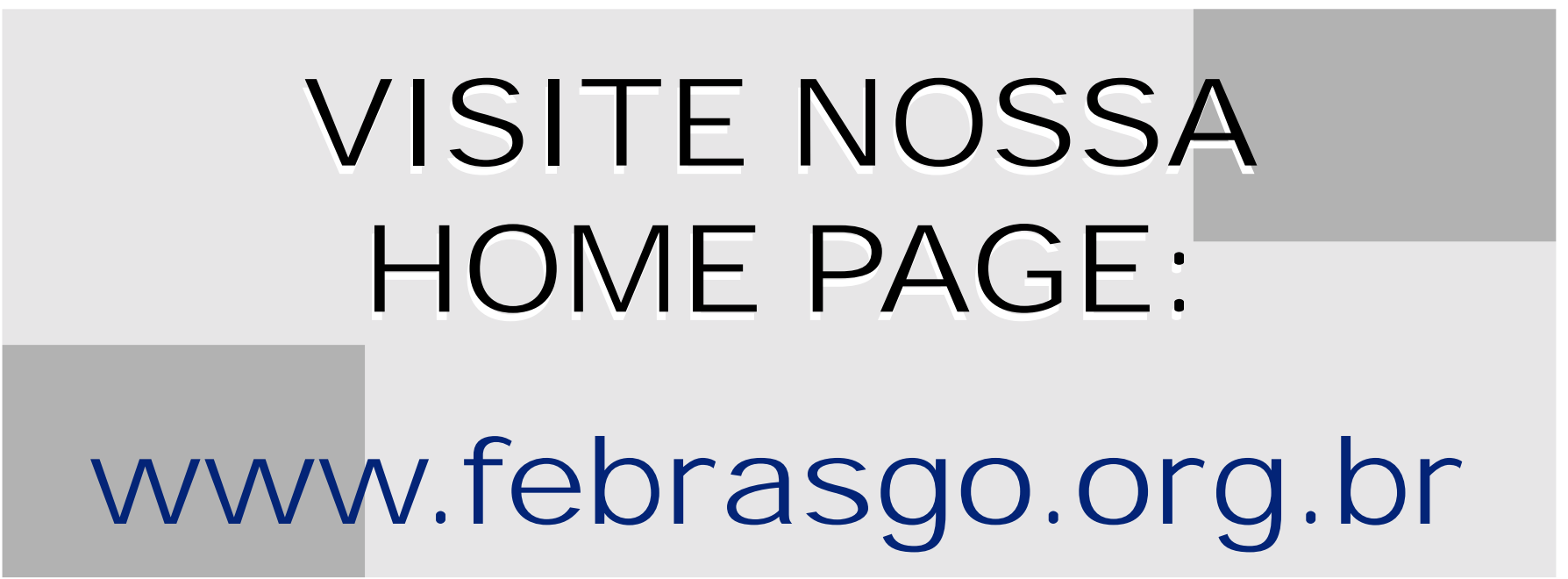

